\section{Developing compact multiphoton systems using femtosecond fiber lasers}

\author{
Shuo Tang, ${ }^{\mathrm{a}, *}$ Jian Liu, ${ }^{\mathrm{b}}$ Tatiana B. Krasieva, ${ }^{\mathrm{c}}$ \\ Zhongping Chen, ${ }^{\mathrm{c}}$ and Bruce J. Tromberg ${ }^{\mathrm{c}}$ \\ aniversity of British Columbia, Department of Electrical \\ and Computer Engineering, 2332 Main Mall, \\ Vancouver, British Columbia, V6T 1 Z4 Canada \\ ${ }^{b}$ PolarOnyx, Inc., 470 Lakeside Drive, Suite F, Sunnyvale, \\ California 94085 \\ 'University of California, Irvine, Beckman Laser Institute, \\ 1002 Health Sciences Road, Irvine, California 92617
}

\begin{abstract}
We implement a fiber-delivered compact femtosecond fiber laser at 1030-nm wavelength in multiphoton imaging. The laser pulse duration is $150 \mathrm{fs}$, the average power is $200 \mathrm{~mW}$, and the repetition rate is $40 \mathrm{MHz}$. The laser measures $200 \times 160 \times 45 \mathrm{~mm}$ in size and its output is delivered through a photonic bandgap fiber. Intrinsic second-harmonic generation signal is excited from rat tail tendon and human skin samples. Two-photon excited fluorescence signal is obtained from human skin tissues stained with exogenous fluorophore. Our results show that femtosecond fiber lasers at 1030-nm wavelength have significant potential in developing compact, all-fiber-based, portable multiphoton systems and endoscopes. () 2009 Society of Photo-Optical Instrumentation Engineers. [DOI: 10.1117/1.3153842]
\end{abstract}

Keywords: multiphoton microscopy; two-photon excited fluorescence; second-harmonic generation; femtosecond fiber lasers.

Paper 08457LR received Dec. 22, 2008; revised manuscript received Mar. 26, 2009; accepted for publication Apr. 22, 2009; published online Jun. 30, 2009.

Multiphoton microscopy (MPM), including two-photon excited fluorescence (TPEF) and second-harmonic generation (SHG), has become an important tool for high-resolution, noninvasive imaging of thick biological tissues. ${ }^{1-3}$ In TPEF, fluorescence from endogenous or exogenous fluorophores is excited by two-photon absorption. SHG is a nonlinear scattering process where two excitation photons are converted into light that is half the wavelength (twice the energy) of the excitation source. TPEF and SHG imaging can provide complementary information about cellular and extracellular matrix structures. ${ }^{4}$

Recently there has been tremendous effort to develop compact multiphoton systems for in vivo imaging and clinical applications. In compact systems, femtosecond pulses from ultrafast lasers are coupled into double cladding photonic crystal fibers $(\mathrm{PCFs})^{5-7}$ or hollow core photonic bandgap fibers $(\mathrm{PBFs}){ }^{8,9}$ Fiber-connected miniature scanning probes are developed to provide flexible access to tissues and acquire MPM images. ${ }^{8-10}$ While the probes are becoming smaller and smaller, the sources that they have used are still the traditional solid-state Ti:sapphire lasers which are bulky, expensive, not portable, and require precise alignment.

*Address all correspondence to: Shuo Tang, E-mail: tang@ece.ubc.ca
Finding new ultrafast laser sources that are low cost, compact, and portable is expected to enhance the impact of MPM in research and particularly in clinical studies. Semiconductor lasers have been recently demonstrated for MPM imaging. ${ }^{11-13}$ By frequency doubling a 1540-nm gainswitched semiconductor laser, Yokoyama et al. have developed a pulsed source with 5-ps pulse duration at 770-nm wavelength, and applied the source for MPM imaging of actin filaments in PtK2 cells. ${ }^{11}$ Using a gain-switched semiconductor laser, Taira et al. developed a 980-nm wavelength source with 3.5-ps pulse duration and obtained MPM images of mouse kidney tissues. ${ }^{13}$ The pulsewidth of those semiconductor lasers is limited to several picoseconds, which cannot excite MPM signals as efficiently as femtosecond pulses.

Recently, femtosecond fiber lasers have emerged as a promising compact light source for MPM applications. ${ }^{14-16}$ Millard et al. used a 1560-nm wavelength femtosecond fiber laser for third-harmonic generation (THG) ${ }^{14}$ Unruh et al. applied a frequency doubled 1560-nm wavelength femtosecond fiber laser for two-photon fluorescence correlation spectroscopy (FCS). ${ }^{15}$ The 1560 -nm femtosecond fiber lasers and their frequency doubled versions were still output into free space. Therefore, they lacked the capability of direct fiber delivery of femtosecond pulses from the laser sources.

Femtosecond fiber lasers at 1030-nm fundamental wavelength have not been reported in MPM applications. There are several advantages of using the 1030-nm wavelength for MPM. Exploring longer wavelength excitation for MPM can potentially reduce tissue scattering and increase imaging depth in turbid tissues. The 1030-nm wavelength band can be used to excite a large selection of fluorescent proteins and dyes that have been originally developed for confocal and fluorescence imaging. ${ }^{13,16,17}$ Squirrell et al. showed that twophoton imaging using 1047-nm excitation wavelength maintained embryo viability over the long term. ${ }^{17}$

In this work, we introduce a compact short-pulsed, highpower, fiber-delivered femtosecond fiber laser at 1030-nm wavelength for MPM applications. The main advantage of using the fiber laser is the compactness of the laser source and fiber delivery of femtosecond pulses, which makes it an ideal source for developing compact and portable MPM systems. Such advantages are demonstrated by coupling the fiber laser to an MPM microscope system to provide excitation wavelength at $1030 \mathrm{~nm}$, and implementing the fiber laser in an all-fiber connected multiphoton spectroscopy system.

The femtosecond fiber laser (PolarOnyx, Sunnyvale, California) is composed of an oscillator that generates seed femtosecond pulses, and an amplifier that boosts the power to $200 \mathrm{~mW}$. In the oscillator, a high concentration Yb-doped PCF is used as the gain medium. A 980-nm pump laser pumps $\mathrm{Yb}$ ions for amplification of intracavity pulses. Passive mode locking is achieved through nonlinear polarization evolution in the slightly birefringent fiber laser cavity. ${ }^{18}$ Polarization filtering is through a fiber-based inline polarization isolator and polarization controllers inside the laser cavity. Amplification of the seed pulse is achieved by using a short piece of high concentration double cladding Yd-doped fiber pumped with another $980-n m$ pump laser. After the amplifier, a piece of PBF is used to compress the pulsewidth. The PBF

1083-3668/2009/14(3)/030508/3/\$25.00 @ 2009 SPIE 




(a)

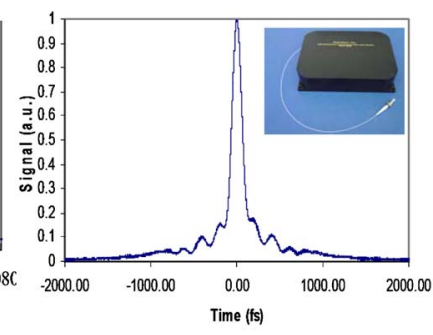

(b)
Fig. 1 Characteristics of the femtosecond fiber laser: (a) spectrum and (b) autocorrelation trace. The insertion shows a photograph of the packaged fiber laser.

can provide $\sim-100 \mathrm{ps} / \mathrm{nm} / \mathrm{km}$ anomalous group velocity dispersion (GVD) to compress positively chirped pulses from the amplifier. The output fiber is terminated with an FC/APC fiber connector to deliver femtosecond pulses.

Figures 1(a) and 1(b) show the spectrum and autocorrelation trace of the laser output. The pulse duration is $150 \mathrm{fs}$ measured at the end of the output fiber, and the spectral bandwidth is $20 \mathrm{~nm}$, which brings the time-bandwidth product of the laser to be $\sim 0.8$. The pulse is not transform limited, and the residue chirp is mainly due to higher order dispersion from the fibers. The laser output power is $200 \mathrm{~mW}$ and pulse repetition rate is $\sim 40 \mathrm{MHz}$. The fiber laser has a highly compact size of $200 \times 160 \times 45 \mathrm{~mm}$, including the oscillator, amplifier, and pump lasers.

Due to its compact size, the fiber laser can be conveniently coupled to any MPM microscope systems to provide excitation wavelengths at $1030 \mathrm{~nm}$. In our experiment, the output of the fiber laser is collimated and coupled into an MPM scanning microscope. Details about the MPM microscope can be found in Ref. 19. Figures 2(a) and 2(b) show the MPM images excited with the 1030-nm wavelength fiber laser. Figure 2(a) shows the backward SHG imaging of rat tail tendon where the collagen fiber bundles are observed. Figure 2(b) shows the TPEF imaging of the cross section (side view) of $e x$ vivo human skin. The skin sample is stained with yelloworange vital nuclear dye, and the cellular structure of the skin is captured by the TPEF imaging. In Fig. 2(b), the TPEF signal is mainly from the epidermis (outer) layer of the skin, which is cellular. The dermis (inner) layer shows little TPEF signal because it is mainly composed of an extracellular ma-
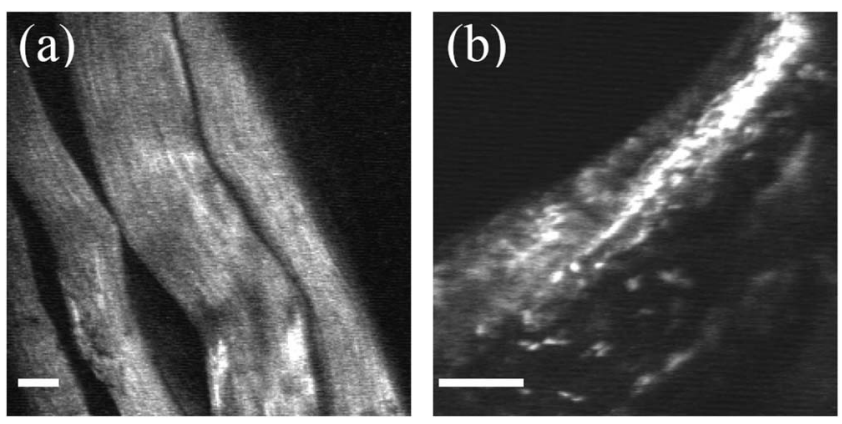

Fig. 2 Multiphoton images excited with the femtosecond fiber laser. (a) SHG image from rat tail tendon. (b) TPEF image from ex vivo human skin stained with nuclear vital dye. The scale bar is $50 \mu \mathrm{m}$.

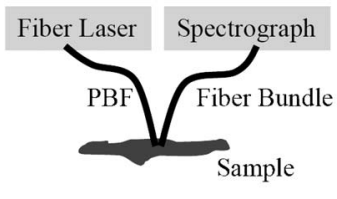

(a)

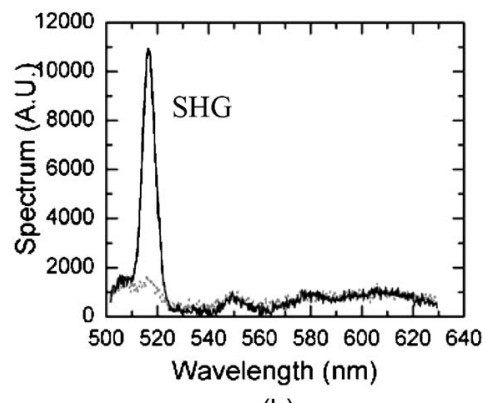

(b)
Fig. 3 (a) Schematic of the MPM spectroscopy system using the fiber laser. (b) MPM spectrum excited from human skin. Solid line: spectrum from dermis layer; doted line: spectrum from epidermis layer.

trix. In this experiment, no dispersion compensation is applied to simplify the system design. Therefore, to minimize pulse broadening of the excitation light, a $10 \times(0.3 \mathrm{NA})$ objective is used that has less dispersion than a high NA objective. The excitation power at the sample location is $\sim 20 \mathrm{~mW}$. The images have $256 \times 256$ pixels, and the acquisition time is $\sim 6$ s/frame. Higher image resolution can be achieved by using a high NA objective and implementing dispersion precompensation to maintain short pulse at the sample location.

To take full advantage of the fiber delivery of femtosecond pulses, the fiber laser is implemented in an all-fiber connected multiphoton spectroscopy system. Figure 3(a) shows the schematic of the experimental setup. Femtosecond pulses from the fiber laser are delivered to the tissue site by PBF. At the end of the PBF, the laser power is $200 \mathrm{~mW}$ and the pulsewidth reaches the minimum of $150 \mathrm{fs}$. Thus, MPM signal can be excited from the proximate area of the end of the excitation fiber. Signal collection is through a fiber bundle positioned next to the excitation fiber. The other side of the fiber bundle is coupled to a spectrograph (SpectraPro-150, Acton Research, Princeton Instruments, Trenton, New Jersey) with a grating of 300 grooves/mm blazed at 500-nm wavelength, followed by a cooled charge-coupled device (CCD) camera (NTE/CCD-512-EBFT, Princeton Instruments, Trenton, New Jersey). Figure 3(b) shows the spectrum of intrinsic MPM signal excited from ex vivo human skin without staining. A 5 -s integration time is used. As shown by the solid line, a strong peak is observed at $515 \mathrm{~nm}$, which corresponds to the SHG signal excited by the $1030-\mathrm{nm}$ wavelength. The strong SHG signal is excited from the dermis layer, composed mostly of collagen fibers. From the epidermis layer, very low SHG signal is observed, as shown by the dotted line. Due to the long excitation wavelength, very low autofluorescence is excited from the skin sample.

Figure 4 shows the comparison of MPM imaging of human skin excited with 1030- and 800-nm wavelengths. The images are obtained using a Zeiss LSM510, where the laser source (Chameleon, Coherent, Santa Clara, California) can be tuned to 1030 and $800 \mathrm{~nm}$, respectively. A $40 \times$ water NA=0.8 objective is used. The laser power before the objective is $\sim 15 \mathrm{~mW}$ in both cases. For the SHG channel, bandpass filters of 500 to $530 \mathrm{~nm}$ and 390 to $465 \mathrm{~nm}$ are used for 1030and $800-\mathrm{nm}$ excitation, respectively. The TPEF channel is filtered with a 535 to $590-\mathrm{nm}$ bandpass filter. Both images are collected in a backward mode. In Fig. 4(a), bright SHG 

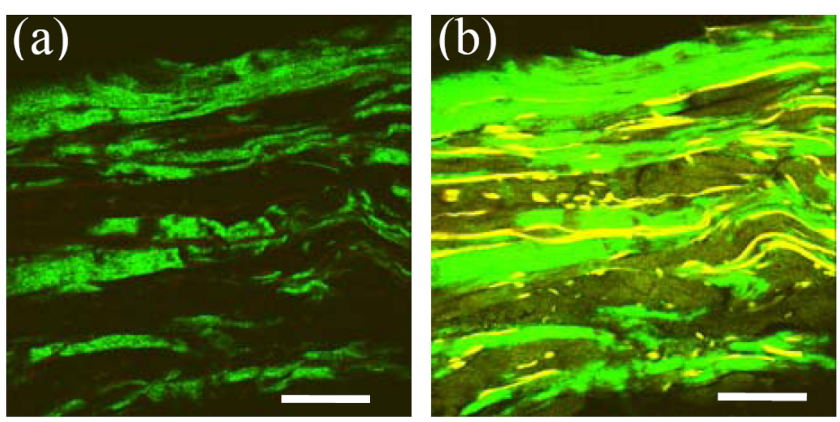

Fig. 4 Comparison of MPM imaging of human skin using 1030- and 800-nm wavelength excitation. (a) Human skin excited by 1030-nm wavelength. (b) The same sample excited by $800-\mathrm{nm}$ wavelength. SHG and TPEF are color coded in green and red, and the overlapping areas are in yellow. Scale bar is $50 \mu \mathrm{m}$.

but very low TPEF signal is observed when 1030-nm excitation is used. In Fig. 4(b), both SHG and TPEF signals are observed from the same sample when $800 \mathrm{~nm}$ excitation is used. In Fig. 4(b), the TPEF signal shows in yellow, because the 390 to $465-\mathrm{nm}$ filter also collects some TPEF signal in the SHG channel. In our study, only a gradual decrease in backward SHG intensity is observed when the excitation moves to longer wavelengths. From 800 to $1030 \mathrm{~nm}$, a $\sim 50 \%$ drop in the SHG intensity is observed, which can be compensated by increasing the laser power by $140 \%$. Therefore, SHG imaging can be implemented using a wide range of laser wavelengths. Figure 4(a) also shows that the autofluorescence background is largely reduced when excitation moves to $1030 \mathrm{~nm}$. This property can be used to provide highly specific fluorescence imaging with no autofluorescence background when used together with exogenous fluorophores.

In conclusion, we have implemented a compact, all-fiberbased femtosecond fiber laser in multiphoton imaging and spectroscopy at the wavelength band of $1030 \mathrm{~nm}$. Optical pulses of $150-\mathrm{fs}$ pulse duration, 200-mW average power, and $40-\mathrm{MHz}$ repetition rate are generated by an all-fiber-based laser system and delivered by a photonic bandgap fiber. The fiber laser is incorporated with a scanning microscopy system to perform multiphoton optical imaging. Our results show that the femtosecond fiber laser can be used to excited intrinsic SHG signals from tissues, and TPEF imaging can be applied in combination with fluorescent staining. An all-fiber connected multiphoton spectroscopy system is developed to demonstrate the potential of femtosecond fiber lasers in developing all-fiber connected portable MPM systems. Overall, femtosecond fiber lasers at 1030 -nm wavelength appear to have excellent potential in applications where all-fiber connected systems are needed, such as multiphoton endoscopes, where the fiber laser can be naturally integrated with a fiber optic probe.

\section{Acknowledgment}

This work was supported by NIH (RR01192) and Air Force Office of Scientific Research.

\section{References}

1. W. Denk, J. H. Strickler, and W. W. Webb, "Two-photon laser scanning fluorescence microscopy," Science 248, 73-76 (1990).

2. W. R. Zipfel, R. M. Williams, and W. W. Webb, "Nonlinear magic: multiphoton microscopy in the biosciences," Nat. Biotechnol. 21, 1368-1376 (2003).

3. P. J. Campagnola, M. D. Wei, A. Lewis, and L. M. Loew, "Highresolution nonlinear optical imaging of live cells by second harmonic generation," Biophys. J. 77, 3341-3349 (1999).

4. A. Zoumi, A. Yeh, and B. J. Tromberg, "Imaging cells and extracellular matrix in vivo by using second-harmonic generation and twophoton excited fluorescence," Proc. Natl. Acad. Sci. U.S.A. 99, 11014-11019 (2002).

5. L. Fu, X. S. Gan, and M. Gu, "Nonlinear optical microscopy based on double-clad photonic crystal fibers," Opt. Express 13, 5528-5534 (2005).

6. L. Fu, A. Jain, C. Cranfield, H. Xie, and M. Gu, "Three-dimensional nonlinear optical endoscopy," J. Biomed. Opt. 12, 040501 (2007).

7. W. Y. Jung, S. Tang, D. T. McCormic, T. Q. Xie, Y. C. Ahn, J. P. Su, I. V. Tomov, T. B. Krasieva, B. J. Tromberg, and Z. P. Chen, "Miniaturized probe based on a microelectromechanical system mirror for multiphoton microscopy," Opt. Lett. 33, 1324-1326 (2008).

8. B. A. Flusberg, J. C. Jung, E. D. Cocker, E. P. Anderson, and M. J. Schnitzer, "In vivo brain imaging using a portable 3.9 gram twophoton fluorescence microendoscope," Opt. Lett. 30, 2272-2274 (2005).

9. C. J. Engelbrecht, R. S. Johnston, E. J. Seibel, and F. Helmchen, "Ultra-compact fiber-optic two-photon microscope for functional fluorescence imaging in vivo," Opt. Express 16, 5556-5564 (2008).

10. M. T. Myaing, D. J. MacDonald, and X. Li, "Fiber-optic scanning two-photon fluorescence endoscope," Opt. Lett. 31, 1076-1078 (2006).

11. H. Yokoyama, H. C. Guo, T. Yoda, K. Takashima, K. Sato, H. Taniguchi, and H. Ito, "Two-photon bioimaging with picosecond optical pulses from a semiconductor laser," Opt. Express 14, 3467-3471 (2006).

12. H. Yokoyama, H. Tsubokawa, H. C. Guo, J. Shikata, K. Sato, K. Takashima, K. Kashiwagi, N. Saito, H. Taniguchi, and H. Ito, "Twophoton bioimaging utilizing supercontinuum light generated by a high-peak-power picosecond semiconductor laser source," J. Biomed. Opt. 12(5), 054019 (2007).

13. K. Taira, T. Hashimoto, and H. Yokoyama, "Two-photon fluorescence imaging with a pulse source based on a 980-nm gain-switched laser diode," Opt. Express 15, 2454-2458 (2007).

14. A. C. Millard, P. W. Wiseman, D. N. Fittinghoff, K. R. Wilson, J. A. Squier, and M. Muller, "Third-harmonic generation microscopy by use of a compact, femtosecond fiber laser source," Appl. Opt. 38, 7393-7397 (1999).

15. J. R. Unruh, E. S. Price, R. G. Molla, R. Q. Hui, and C. K. Johnson, "Evaluation of a femtosecond fiber laser for two-photon fluorescence correlation spectroscopy," Microsc. Res. Tech. 69, 891-893 (2006).

16. J. R. Unruh, E. S. Price, R. G. Molla, L. Stehno-Bittel, C. K. Johnson, and R. Q. Hui, "Two-photon microscopy with wavelength switchable fiber laser excitation," Opt. Express 14, 9825-9831 (Oct. 2006).

17. J. M. Squirrell, D. L. Wokosin, J. G. White, and B. D. Bavister, "Long-term two-photon fluorescence imaging of mammalian embryos without compromising viability," Nat. Biotechnol. 17, 763-767 (1999).

18. M. Hofer, M. E. Fermann, F. Haberl, M. H. Ober, and A. J. Schmidt, "Mode locking with cross-phase and self-phase modulation," Opt. Lett. 16, 502-504 (1991).

19. S. Tang, T. B. Krasieva, Z. P. Chen, and B. J. Tromberg, "Combined multiphoton microscopy and optical coherence tomography using a 12-fs broadband source," J. Biomed. Opt. 11(2), 020502 (2006). 